\title{
OBSIDIANAS EN EL BOSQUE: DETERMINACIÓN GEOQUÍMICA DE ARTEFACTOS ARQUEOLÓGICOS DEL S-O DE RÍO NEGRO Y N-O DE CHUBUT (PATAGONIA ARGENTINA) ${ }^{1}$
}

\author{
OBSIDIAN IN THE FOREST: GEOCHEMISTRY OF ARCHAEOLOGICAL \\ ARTIFACTS FROM SW RÍO NEGRO AND NW CHUBUT (PATAGONIA, \\ ARGENTINA)
}

\author{
Cristina Bellelli², Mariana Carballido Calatayud ${ }^{3}$ y Charles Stern ${ }^{4}$
}

\begin{abstract}
Presentamos nuevos datos acerca de la caracterización geoquímica de artefactos de obsidiana de sitios arqueológicos ubicados en el bosque mixto de Nothofagus y Austrocedrus del noroeste de la Patagonia, Argentina. Estos datos se suman a la información disponible para esta área, permitiéndonos discutir la distribución espacial y la circulación de obsidiana en el interior del bosque y entre este ambiente y la estepa, al este, y la costa del Pacífico, al oeste. Analizamos por ICP-MS la composición geoquímica de 32 artefactos arqueológicos de obsidiana de siete sitios ubicados en las localidades de El Manso, El Hoyo y Cholila. Estos datos indican la existencia de un eje de circulación norte-sur de obsidiana en el interior del bosque y otro eje de circulación este-oeste entre la estepa y el bosque a distancias considerables. No se ha encontrado obsidiana procedente de fuentes ubicadas en Chile. Los cazadores-recolectores comenzaron a habitar el bosque andino patagónico en el Holoceno Temprano. Sin embargo, hasta la realización de nuestro análisis no había registro del uso de la obsidiana dentro del ambiente de bosque hasta el Holoceno Tardío, cuando ocurrió la ocupación humana más intensa de estos espacios. Estos nuevos datos extienden la discusión temporal del uso de la obsidiana en el bosque al Holoceno Temprano.
\end{abstract}

Palabras claves: bosque mixto, obsidiana, procedencia, geoquímica, ICP-MS.

We present new data about the trace-element geochemistry of obsidian artifacts from archaeological sites located in the mixed Nothofagus and Austrocedrus forest of northwest Patagonia, Argentina. These data enlarge the available information in this field and allow us to discuss both the spatial distribution and the circulation of obsidian inside the forest and between this environment and both the steppes to the east and the Pacific coast to the west. We analyzed by ICP-MS the trace-element composition of 32 archaeological obsidian artifacts from seven sites located in the localities of El Manso, El Hoyo and Cholila. These data indicate north-south circulation of obsidian within the interior of the forest, and also east-west circulation over considerable distances between the steppes and the forest. No obsidian from sources in Chile has been found. Even though hunter-gatherers began to inhabit the Andean Patagonian forest in the Early Holocene, previous studies have not recorded the use of obsidian inside the forest environment until Late Holocene when the most intense human occupation of these spaces occurred. The new data presented here extends the temporal discussion of the use of obsidian in the forest back into the Early Holocene.

Key words: Forest, obsidian, provenance, geochemistry, ICP-MS.

Las numerosas investigaciones que desde hace casi treinta años se desarrollan en el bosque continental patagónico han permitido modelar distintas formas de ocupación y uso humano de este ambiente a lo largo del Holoceno. De modo general hay coincidencia en proponer que este uso debió implicar algún tipo de complementariedad ambiental con otros entornos más productivos, como el ecotono, la estepa o el litoral pacífico debido a las características del bosque (Arrigoni 1997; Aschero et al. 2005; Belardi y Campán 1999; Borrero y Muñoz 1999; Espinosa 2000, 2002; Goñi 1988; Hajduk et al. 2011; Silveira 1987, 1999; entre otros).

El bosque continental patagónico desarrolla un paisaje modelado por la orogenia andina y la acción glaciar que dio como resultado una topografía

\footnotetext{
1 Una primera versión de este artículo fue presentada en el simposio "Desde las fuentes: estudios de selección y aprovisionamiento de minerales. Implicancias en la producción lítica, alfarera, metalúrgica y de manifestaciones rupestres”, en el marco del XIX Congreso Nacional de Arqueología Argentina, Tucumán (agosto 2016). Este manuscrito fue evaluado por pares externos y editado por el Comité Editorial de Chungara y los editores invitados Ariel D. Frank, Fabiana Skarbun y Manuel E. Cueto.

2 CONICET - Instituto Nacional de Antropología y Pensamiento Latinoamericano, Universidad de Buenos Aires, Buenos Aires, Argentina. crisbellelli@yahoo.com.ar

3 CONICET - Instituto Nacional de Antropología y Pensamiento Latinoamericano, Universidad de Buenos Aires, Buenos Aires, Argentina.mcarballidocalata@hotmail.com

$4 \quad$ Universidad de Colorado, Boulder, Colorado. EE.UU. Charles.Stern@ colorado.edu
}

Recibido: febrero 2017. Aceptado: febrero 2018.

http://dx.doi.org/10.4067/S0717-73562018005000601. Publicado en línea: 6-abril-2018. 
quebrada y numerosos valles. Se extiende a lo largo de una estrecha franja de $2.000 \mathrm{~km}$ de largo y $120 \mathrm{~km}$ de ancho promedio que presenta una gran heterogeneidad de tipos de bosque, suelos y regímenes de perturbación (Armesto et al. 1995). Los patrones regionales de vegetación reflejan gradientes climáticos N-S y O-E que producen una disminución en la riqueza de especies vegetales y en la densidad y diversidad de mamíferos hacia el S y el E (Arroyo et al. 1995).

En el área de estudio, comprendida entre el límite sur del Parque Nacional Nahuel Huapi y el límite norte del Parque Nacional Los Alerces y entre la frontera con Chile y el meridiano de $71^{\circ} \mathrm{O}$ (Figura 1), el gradiente de precipitaciones y el relieve determinan el desarrollo de distintas configuraciones de vegetación. En el extremo O (Parque Nacional Lago Puelo), donde las precipitaciones promedio son de $1600 \mathrm{~mm}$, se encuentran especies propias del bosque valdiviano chileno (bosque lluvioso siempre verde), y a medida que se avanza al $\mathrm{E}$ se desarrolla plenamente el bosque mixto de Nothofagus y Austrocedrus (Faggi 1994). Este es el ambiente que domina en nuestra área de investigación cuya configuración responde también a ciertas perturbaciones naturales vinculadas con la acción del volcanismo y del fuego (Veblen et al. 1992, 1995).
En este contexto, pueden reconocerse al menos tres de las particularidades que presentan desafíos a una forma de vida cazadora - recolectora en el bosque, la escasez de presas de alto rinde, la topografía irregular y el importante desarrollo de la vegetación que dificulta el tránsito y la visibilidad de los recursos de este ambiente (Arrigoni 1997; Bellelli et al. 2003; Carballido Calatayud 2009; Carballido Calatayud y Fernández 2013; Hajduk et al. 2004; Lezcano et al. 2010; Scheinsohn 2004; Silveira 1999). Específicamente para el Holoceno Tardío, se han propuesto tres modalidades de uso del bosque que contemplan la existencia de estrategias de amplia movilidad entre el bosque y otros entornos, tanto sea (a) que los grupos permanecieran más tiempo en la estepa o el ecotono que en el bosque (Arrigoni 1997; Silveira 1999; entre otros), (b) que residieran de manera semipermanente en el bosque integrando el ecotono de manera regular (Albornoz y Hajduk 2006; Hajduk et al. 2004; Lezcano et al. 2010; entre otros) o, finalmente, (c) la posibilidad de grupos adaptados al bosque (Pérez y Smith 2008; Pérez 2010). Estas propuestas no son excluyentes y pueden haberse desarrollado de manera complementaria en distintos momentos y espacios ya que deben considerarse como parte de la gama de estrategias posibles de ser implementadas

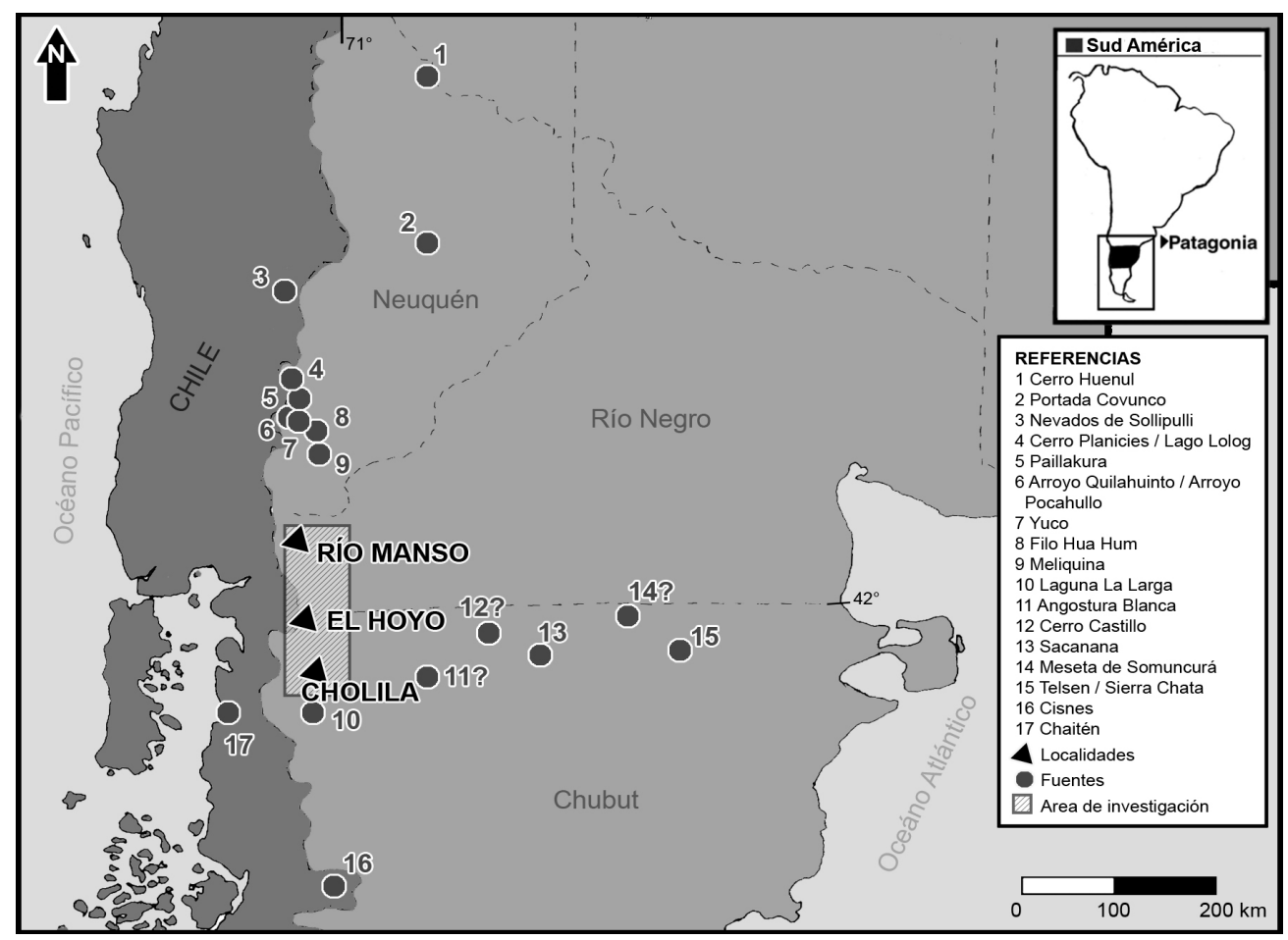

Figura 1. Localidades arqueológicas bajo estudio y fuentes de obsidiana conocidas. Archaeological localities under study and known obsidian sources. 
dentro del bosque patagónico (Carballido Calatayud 2009). La información generada en la última década ha llevado la ocupación humana en nuestra área de investigación y en áreas vecinas al Holoceno Temprano y Medio (Bellelli et al. 2013; Hajduk et al 2006; Lezcano et al. 2010). Esta ampliación de la escala temporal plantea la posibilidad de nuevas respuestas humanas a la ocupación del bosque en momentos más tempranos.

Tanto para la reformulación de los modelos de ocupación humana del bosque en momentos tardíos, como para la elaboración de propuestas que expliquen el modo en que se ocupó en momentos más tempranos, es clave el tema de la circulación o el tránsito desde/hacia otros ambientes (el ecotono próximo, la estepa y el litoral pacífico), a lo largo de la cordillera, conectando ambientes boscosos diferentes y en el interior del bosque.

En la mayoría de los contextos arqueológicos conocidos en el bosque norpatagónico se ha advertido que existe un uso complementario de materias primas líticas provenientes de distintos ambientes, algunos muy alejados entre sí (Hajduk et al. 2004; López et al. 2010; Silveira 1996). En ese sentido, es necesario explorar las distintas modalidades de obtención de los recursos líticos. Así, los artefactos de obsidiana, por sus características geoquímicas, permiten establecer, dentro de límites estadísticos, potenciales fuentes de abastecimiento y procedencia utilizadas en el pasado. De esta forma se puede acceder al conocimiento de la dinámica de ocupación humana de los diferentes espacios de la Patagonia, informando sobre aspectos relacionados con la movilidad de los grupos humanos, la circulación y el uso de esta materia prima lítica en diferentes ambientes separados por distancias variables (Bellelli et al. 2006).

En este trabajo presentamos nuevos datos sobre la caracterización geoquímica de artefactos de obsidiana provenientes de sitios arqueológicos ubicados exclusivamente en el bosque mixto de Nothofagus y Austrocedrus, en el valle del río Manso inferior (provincia de Río Negro) y en las localidades El Hoyo y Cholila (provincia de Chubut) (Figura 1). Esta información se pondrá en contexto a partir de su comparación con los resultados obtenidos en trabajos previos, en los que se determinaron distintas fuentes. Así, ampliamos la base de datos disponible, ya que hasta el momento sólo contábamos con datos de la localidad Cholila (Bellelli y Pereyra 2002; Bellelli et al. 2006). Además, comparamos los nuevos resultados con la información disponible sobre fuentes de aprovisionamiento del norte y centro de Patagonia (provincias de Neuquén, Río Negro y Chubut, Argentina, y también de Chile en las mismas latitudes) (entre otros, Bellelli et al. 2006; López et al. 2009; Méndez et al. 2008-2009; Pérez et al. 2015; Stern et al. 2000; Stern et al. 2002, 2009). Esto nos permite, por un lado, discutir la distribución espacial de este material y su circulación en el interior del bosque y entre este ambiente, la estepa y el litoral pacífico. Por otro lado, estos resultados posibilitan explorar con un mayor grado de detalle la profundidad temporal del uso de obsidianas en el bosque.

\section{Estado del Conocimiento}

Hasta el momento contábamos con resultados geoquímicos para el sector sur de nuestra área de investigación que se extiende desde el límite meridional del Parque Nacional Nahuel Huapi hasta el límite septentrional del Parque Nacional Los Alerces y desde la frontera con Chile hasta la zona ecotonal y de estepa que se distribuye a lo largo del meridiano de $71^{\circ} \mathrm{O}$. Dichos resultados provienen del sitio estratificado Cerro Pintado, de tres conjuntos de superficie a cielo abierto: Juncal de Calderón 1, Juncal de Calderón 3 y Los Guanacos 3 y de un hallazgo aislado, todos en la localidad Cholila (Chubut), ubicados a unos $11 \mathrm{~km}$ lineales entre sí (Bellelli et al. 2006). Las muestras arqueológicas previamente analizadas indican el uso de tres fuentes: Sacanana (S1), fuente secundaria localizada en el cerro Guacho en la meseta de Somuncurá, que es una de las más ampliamente utilizadas en el norte y centro patagónico y que fuera descripta por primera vez por Stern et al. 2000; Portada Covunco (PC), fuente secundaria ubicada en el curso del arroyo homónimo, con una alta presencia en sitios de Neuquén, Río Negro y Chubut, así como en un sitio ubicado en el actual territorio chileno, a escasos $70 \mathrm{~km}$ de la fuente (Alberti et al. 2016; Bellelli et al. 2006; Boschín y Massaferro 2014; Fernández y Vítores 2015; López et al. 2009; Pérez et al. 2015; Stern et al. 2009; Stern et al. 2012) y, finalmente, una fuente cercana a Cholila, Laguna La Larga (LL), ubicada a orillas de la laguna homónima, adyacente al límite sureste del Parque Nacional Los Alerces. En este caso se localizó un afloramiento de mala calidad para la talla y pequeños guijarros de buena calidad. Esta fuente parece haber sido utilizada de manera muy poco frecuente, a escala local o de regiones vecinas (Arrigoni 2005; Bellelli et al. 2006).

Sin embargo, la cantidad de fuentes conocidas para el norte de Patagonia no se reduce a estas tres. De hecho, el trabajo de numerosos colegas durante los últimos diez años nos muestra un panorama más complejo, que sintetizamos a continuación, de norte a sur, para las provincias de Neuquén, Río Negro y Chubut y para Chile en latitudes equivalentes (Figura 1): 
- Cerro Huenul-Neuquén (CHN), fuente primaria ubicada al norte de dicha provincia, en la margen derecha del río Colorado (Durán et al. 2004);

- Cerro de las Planicies/Lolog-Neuquén (CP/ LL), fuente primaria en la cima del Cerro de las Planicies y secundaria en la costa norte del lago Lolog (Parque Nacional Lanín) (López et al. 2009; Pérez et al. 2015);

- Arroyo Quilahuinto y Arroyo Pocahullo Neuquén (QU/AP) fuentes secundarias ubicadas en dos arroyos que desembocan en el lago Lácar, en las cercanías de San Martín de los Andes (López et al. 2009; Pérez et al. 2015);

- Filo HuaHum-Neuquén (FHH) fuente secundaria en un curso de agua que desemboca en el lago del mismo nombre (Pérez et al. 2015);

- Paillakura - Neuquén (PK) (ex Desconocida 1 de López et al. 2009), fuente primaria ubicada en el sector centro oriental del cordón Chapelco (Pérez et al. 2015); - Meliquina - Neuquén (MQ) (ex Desconocida 2 de López et al. 2009), fuente secundaria de rodados aislados en la costa oeste del lago Meliquina y en la cuenca del río homónimo (Pérez et al. 2015);

- Yuco-Neuquén (YC), fuente secundaria en una playa cercana a la localidad de Yuco, en la costa centro-norte del lago Lácar (López et al. 2009; Pérez et al. 2015);

- Telsen/Sierra Chata I y II, en Sierra Negra (meseta de Somuncurá-Chubut), fuente secundaria al noroeste de Telsen, a partir de la cual se habían definido dos subtipos (T/SCI, T/SCII - Stern et al. 2000) aunque luego se determinó que el subtipo T/ SCII no correspondería a esta fuente (Gómez Otero y Stern 2005);

- Meseta de Somuncurá-Río Negro o Chubut (MS1), identificada a partir de artefactos arqueológicos, de fuente aún desconocida aunque se presume que está situada en el corazón de dicha meseta (Gómez Otero y Stern 2005);

- Cerro Castillo-Río Negro o Chubut (CC), también identificada a partir de artefactos arqueológicos de fuente desconocida, sobre la margen suroeste de la meseta de Somuncurá (Stern et al. 2000);

- Angostura Blanca- Chubut (AB), identificada a partir de artefactos arqueológicos y de rodados de muy mala calidad para la talla presentes en el valle del río Chubut y sus tributarios (área Piedra Parada) (Bellelli et al. 2006);

- Chaitén (CH 1), fuente primaria, en las proximidades del volcán homónimo en el centrosur de Chile, en la Región de Los Lagos (Stern y Curry 1995; Stern et al. 2002);

- Nevados de Sollipulli (MEL), fuente primaria, al oeste del volcán Sollipulli en el centro-sur de Chile, Región de la Araucanía (Stern et al. 2008);
- Cisnes (CIS), identificada a partir de artefactos arqueológicos y de rodados de mala calidad ubicados en las cabeceras del río homónimo, Región de Aisén (Chile) (Méndez et al. 20082009).

Estas diferentes fuentes pueden dividirse entre aquellas relacionadas con los volcanes calco-alcalinos activos de los Andes altos (Chaitén y Nevados de Sollipulli), los relacionados con los centros volcánicos Plioceno-Pleistocénicos ligeramente alcalinos del este de los Andes altos (Portada Covunco, Cerro Huenul, Cerro de las Planicies/ Lolog, Arroyo Quilahuinto y Arroyo Pocahullo, Filo HuaHum, Paillakura, Meliquina, Yuco, Laguna La Larga) y, finalmente, los centros Miocénicos altamente alcalinos asociados con las lavas de retro-arco de la meseta Patagónica (Sacanana, Cerro Castillo, Telsen/Sierra Chata y la Meseta de Somuncurá).

\section{Las Obsidianas en los Conjuntos Arqueológicos del Bosque}

Una característica recurrente en los conjuntos líticos del bosque es la baja frecuencia de obsidiana. En la Tabla 1 se presentan los valores de la totalidad de la muestra lítica y los de esta materia prima para cada uno de los sitios analizados. Los instrumentos de obsidiana, además de ser muy escasos, en su gran mayoría están agotados, fracturados y sin posibilidad de continuar siendo mantenidos, o sea que han llegado al término de su vida útil, como sucede con la mayoría de las piezas excepto en dos casos en que se combinan más de un filo o punta. Los grupos tipológicos representados se limitan a las puntas de proyectil (un fragmento de limbo y uno de pedúnculo), preforma sobre lasca (una), puntas burilantes (dos, una de ellas doble y la otra acompañada por tres filos naturales con rastros complementarios), perforador (un fragmento no diferenciado), muesca (un fragmento no diferenciado), artefacto de formatización sumaria (uno) y fragmentos no diferenciados de artefactos o filos (cinco). Esta enumeración sugiere una tendencia al uso de esta materia prima para confeccionar instrumentos con puntas activas en desmedro de los filos o bordes activos, de los que sólo están representados fragmentos cuyo grupo tipológico no puede reconocerse o bien filos naturales con rastros complementarios.

Por otro lado, las dimensiones de todos los artefactos de obsidiana son muy reducidas. Por ejemplo, en los desechos de talla, los largos de las piezas enteras no superan los $3,5 \mathrm{~cm}$ y el rango 
dominante de esta medida está entre los 0,5 y los 0,99 $\mathrm{cm}$, que en los sitios estratificados supera la mitad de la muestra. Así, en Paredón Lanfré el 57,14\% de los desechos enteros está dentro de este rango, en Población Anticura el 66,67\%, en Risco de Azócar 1 $72,73 \%$ y en Cerro Pintado 54,37\%. En los dos sitios de superficie, Los Guanacos 3 y 4, este rango tiene una menor representación $(33,33 \%)$ debido a las técnicas de recolección que no incluyeron el uso de zaranda. Por otra parte, el índice de corteza en todas las muestras de artefactos de obsidiana no supera el 13\%. Entre los instrumentos, solo en Cerro Pintado hay uno con reserva de corteza mientras que en el resto de los conjuntos no hay instrumentos con corteza en esta materia prima. Entre los desechos de talla, el porcentaje de piezas de obsidiana con corteza ronda entre el 0 y el $13 \%$. El único núcleo en esta materia prima no presenta reserva de corteza, señal también de su estado agotado. La escasa representatividad de esta variable en los desechos de obsidiana es también una característica de los desechos de otras materias primas $\mathrm{y}$, junto con lo apuntado sobre los rangos de tamaños y la alta frecuencia de desechos angulares, y la presencia de desechos de reducción bifacial y de reactivación, indican que predominaban las actividades de formatización de filos, mantenimiento y reactivación.

En síntesis, debido a la poca presencia de obsidiana en las muestras, al tamaño muy pequeño de la mayoría de los desechos de esta materia prima y a que el análisis geoquímico requiere que las piezas tengan un peso superior a 0,15 gr la cantidad que pudo ser analizada se vio limitada ${ }^{1}$.

\section{Materiales y Métodos}

De las 34 piezas seleccionadas, 33 son desechos de talla y una es el núcleo mencionado en la Tabla 1. Por las características del conjunto instrumental y por las limitaciones apuntadas más arriba, elegimos como criterio de conservación del material sólo analizar desechos de talla y el núcleo, preservando a los instrumentos. El análisis determinó que dos desechos no son de obsidiana, por lo que el número final acá presentado es de 31 desechos y un núcleo².

Dichos artefactos provienen de tres localidades:

- Valle del río Manso inferior (en adelante El Manso): en el extremo norte del área, donde determinamos 11 desechos de talla de dos sitios. Población Anticura es un sitio estratificado con arte rupestre del que provienen seis muestras, de las cuales aquí se consideran solo cuatro, porque dos de ellas no se caracterizaron como obsidianas, tal como se dijo precedentemente. La secuencia de ocupación fue datada ${ }^{3}$ entre el $8.230 \pm 110$ a.p. LP-2758 y $280 \pm 40$ a.p. LP-2413 (Bellelli et al. 2013; Fernández et al. 2013). Tres de los desechos analizados fueron recuperados en los niveles más modernos (entre $700 \pm 60$ a.p. LP-2084 y $280 \pm 40$ a.p. LP-2413) y uno en el nivel basal de la excavación. En Paredón Lanfré se analizaron cinco muestras distribuidas a lo largo de toda la secuencia de ocupación que fue datada entre $1.500 \pm 60$ a.p. LP-1429 y $330 \pm 50$ a.p. LP-1587. Dos de los desechos provienen de los niveles más modernos, mientras que el resto se ubica entre $1.500 \pm 60$ a.p. LP-1429y $790 \pm 60$ a.p. LP-1607 (Bellelli et al. 2007).

Tabla 1. Frecuencia artefactos de obsidiana de los sitios analizados.

Frequency of obsidian artifacts in analyzed archaeological sites.

\begin{tabular}{|c|c|c|c|c|c|c|c|c|c|}
\hline \multirow{2}{*}{ Localidad } & \multirow{2}{*}{ Sitio } & \multicolumn{2}{|c|}{ Instrumentos } & \multicolumn{3}{|c|}{ Desechos } & \multicolumn{3}{|c|}{ Núcleos } \\
\hline & & Total & $\begin{array}{c}\text { Total de } \\
\text { obsidiana }\end{array}$ & Total & $\begin{array}{c}\text { Total de } \\
\text { obsidiana }\end{array}$ & Analizados & Total & $\begin{array}{c}\text { Total de } \\
\text { obsidiana }\end{array}$ & Analizados \\
\hline \multirow{2}{*}{ El Manso } & $\begin{array}{l}\text { Paredón } \\
\text { Lanfré }\end{array}$ & 38 & $1(2,63 \%)$ & 1454 & $30(2,06 \%)$ & $5(16,66 \%)$ & 5 & - & - \\
\hline & $\begin{array}{c}\text { Población } \\
\text { Anticura }\end{array}$ & 64 & $1(1,56 \%)$ & $429 *$ & $32(7,45 \%)$ & $4(12,5 \%)$ & 4 & - & - \\
\hline El Hoyo & $\begin{array}{l}\text { Risco de } \\
\text { Azócar } 1\end{array}$ & 9 & - & 563 & $31(5,5 \%)$ & - & 1 & $1(100 \%)$ & $1(100 \%)$ \\
\hline \multirow{3}{*}{ Cholila } & $\begin{array}{c}\text { Los } \\
\text { Guanacos } 3\end{array}$ & 42 & $1(4 \%)$ & 918 & $27(2,94 \%)$ & $5(18,51 \%)$ & 10 & - & - \\
\hline & $\begin{array}{c}\text { Los } \\
\text { Guanacos } 4\end{array}$ & 8 & $1(12,5 \%)$ & 145 & $10(7,23 \%)$ & $2(20 \%)$ & 3 & - & - \\
\hline & $\begin{array}{c}\text { Cerro } \\
\text { Pintado }\end{array}$ & 76 & $\begin{array}{c}11 \\
(10,47 \%)\end{array}$ & 7286 & $\begin{array}{c}230 \\
(3,16 \%)\end{array}$ & $15(6,52 \%)$ & 10 & - & - \\
\hline
\end{tabular}

* El análisis de desechos está en curso. Esta cantidad refleja los analizados hasta ahora. 
- El Hoyo: valle del río Epuyén, en el alero estratificado con arte rupestre Risco de Azocar 1 las ocupaciones humanas se fecharon entre $1.690 \pm 60$ a.p. LP-1575 y $820 \pm 60$ a.p. LP-1665. De aquí analizamos un núcleo agotado recuperado en un nivel comprendido entre $1.690 \pm 60$ a.p. LP-1575 y $1.250 \pm 70$ a.p. LP-1557 (Podestá et al. 2007).

- Cholila: en el extremo sur del área, donde analizamos siete desechos de talla recuperados en dos sitios de superficie (cinco en Los Guanacos 3 y dos en Los Guanacos 4) y 15 del alero estratificado con arte rupestre Cerro Pintado que tiene cuatro fechados

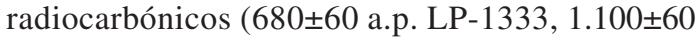
a.p. LP-1439, $1.120 \pm 60$ a.p. LP-1427 y $1.870 \pm 80$ a.p. LP-1313 (Bellelli et al. 2003). Dos de las muestras analizadas en Cerro Pintado provienen del nivel fechado en $680 \pm 60$ a.p. LP-1333 mientras que el resto se recuperó en niveles superiores, sin datar.

En la Tabla 2 se sintetiza esta información, las características de cada sitio y la descripción de la muestra. El análisis se hizo en el Departamento de Servicios Geológicos de la Universidad de Colorado. Las muestras fueron molidas a un polvo muy fino que fue disuelto en ácido fluorhídrico. Posteriormente, fueron analizadas por medio de ICPMS (espectrometría de masas por plasma iónicamente acoplado) para obtener la composición de elementos traza en partes por millón (ppm). Los análisis de los estándares internos indican que la precisión para todos los elementos es mayor al $\pm 10 \%$. Los resultados obtenidos fueron comparados con aquellos que se disponen para muestras geológicas de las fuentes de Patagonia norte y central.

\section{Resultados}

Los resultados de los elementos traza y las tierras raras expuestos en las Tablas 3 y 4 y expresados en $\mu \mathrm{g} \mathrm{g}^{-1}$ indican que tres de las muestras-CP2 de Cholila y PA 4 y 5 de El Manso -no son obsidianas-, por lo tanto el total que se presenta aquí se reduce a 32. En total se identificaron muestras procedentes

Tabla 2. Características de los sitios y descripción de las muestras. Sites characteristics and samples description.

\begin{tabular}{|c|c|c|c|c|c|}
\hline Localidad & Sitio & Tipo de sitio & $\begin{array}{l}\mathrm{N}^{\circ} \text { muestra } \\
\text { laboratorio }\end{array}$ & Cronología ${ }^{14} \mathrm{C}$ & Descripción \\
\hline \multirow{9}{*}{ El Manso } & \multirow{4}{*}{$\begin{array}{c}\text { Población } \\
\text { Anticura (PA) }\end{array}$} & \multirow{4}{*}{ estratificado } & PA6 & $480 \pm 70$ a.p. (Fernández et al.2013) & Roja, fractura concoidal \\
\hline & & & PA1 & $700 \pm 60$ a.p. (Fernández et al.2013) & Negra, fractura concoidal \\
\hline & & & PA2 & $\mathrm{s} / \mathrm{f}$ & Negra, fractura concoidal \\
\hline & & & PA3 & $8.230 \pm 110$ a.p. $-8.010 \pm 130$ a.p. (Bellelli et al. 2013) & Negra grisácea, fractura concoidal \\
\hline & \multirow{5}{*}{$\begin{array}{c}\text { Paredón } \\
\text { Lanfré (PL) }\end{array}$} & \multirow{5}{*}{ estratificado } & PL3 & \multirow{2}{*}{ Post $490 \pm 60$ a.p. (Bellelli et al. 2007) } & Negra, fractura concoidal \\
\hline & & & PL4 & & Negra, fractura concoidal \\
\hline & & & PL5 & Entre $790 \pm 60$ a.p. y $1500 \pm 60$ ap (Bellelli et al. 2007) & Negra, fractura concoidal \\
\hline & & & PL1 & $1.480 \pm 70$ a.p. (Bellelli et al. 2007) & Negra, fractura concoidal \\
\hline & & & PL2 & $1.500 \pm 60$ a.p. (Bellelli et al. 2007) & Marrón, fractura concoidal \\
\hline El Hoyo & $\begin{array}{c}\text { Risco de Azócar } \\
1 \text { (RA1) }\end{array}$ & estratificado & RA1-1 & $1.690 \pm 60$ a.p. (Podestá et al. 2007) & Negra, fractura concoidal \\
\hline \multirow{22}{*}{ Cholila } & \multirow{5}{*}{$\begin{array}{c}\text { Los Guanacos } 3 \\
\text { (LG3) }\end{array}$} & \multirow{5}{*}{ superficie } & LG3-5 & \multirow{5}{*}{$\mathrm{s} / \mathrm{f}$} & Negra, fractura concoidal \\
\hline & & & LG3-1 & & Negra, friable \\
\hline & & & LG3-2 & & Negra grisácea, fractura concoidal \\
\hline & & & LG3-4 & & Negra, opaca, fractura concoidal \\
\hline & & & LG3-3 & & Negra, opaca, con impurezas, mala fractura \\
\hline & \multirow{2}{*}{$\begin{array}{c}\text { Los Guanacos } 4 \\
\text { (LG4) }\end{array}$} & \multirow{2}{*}{ superficie } & LG4-1 & \multirow{2}{*}{$\mathrm{s} / \mathrm{f}$} & Negra, traslúcida, fractura concoidal \\
\hline & & & LG4-2 & & Negra, opaca, fractura concoidal \\
\hline & \multirow{15}{*}{$\begin{array}{l}\text { Cerro Pintado } \\
\text { (CP) }\end{array}$} & \multirow{15}{*}{ estratificado } & $\mathrm{CP} 1$ & \multirow{11}{*}{ Post $680 \pm 60$ a.p. (Bellelli et al. 2003) } & Negra, fractura concoidal \\
\hline & & & $\mathrm{CP} 3$ & & Negra, fractura concoidal \\
\hline & & & $\mathrm{CP} 4$ & & Negra, fractura concoidal \\
\hline & & & CP5 & & Negra, fractura concoidal \\
\hline & & & CP6 & & Negra, friable \\
\hline & & & $\mathrm{CP} 7$ & & Negra, fractura concoidal \\
\hline & & & CP8 & & Gris oscura, traslúcida, fractura concoidal \\
\hline & & & CP9 & & Negra, friable \\
\hline & & & $\mathrm{CP} 10$ & & Negra, fractura concoidal \\
\hline & & & CP11 & & Negra, fractura concoidal \\
\hline & & & $\mathrm{CP} 12$ & & Negra, traslúcida, fractura concoidal \\
\hline & & & CP13 & $680 \pm 60$ a.p. (Bellelli et al. 2003) & Negra, fractura concoidal \\
\hline & & & CP14 & Post $680 \pm 60$ a.p. (Bellelli et al. 2003) & Negra con bandas grises, fractura concoidal \\
\hline & & & $\mathrm{CP} 15$ & $680 \pm 60$ a.p. (Bellelli et al. 2003) & Negra, fractura concoidal \\
\hline & & & CP16 & Post $680 \pm 60$ a.p. (Bellelli et al. 2003) & Negra, fractura concoidal \\
\hline
\end{tabular}


de 10 fuentes distintas, complejizando el panorama que teníamos hasta el momento (Tabla 5 y Figura 2). En El Manso se identificó el uso de al menos cinco fuentes diferentes: S1 (Sacanana 1), MS1 (Meseta de Somuncurá 1), PC1 (Portada Covunco), PK (Paillakura) y QU/AP (Quilahuinto/Pocahullo) (Figura 2A). La única muestra recuperada en El Hoyo que pudo ser analizada proviene de S1 (Figura 2B). En los sitios de Cholila se identificaron siete fuentes diferentes: S1, LL (Laguna La Larga), CC (Cerro Castillo), CP/LL1 (Cerro Planicies/Lago Lolog), YC (Yuco), PK y una desconocida hasta el momento (Figura 2C).

En el caso que estamos presentando acá, prácticamente el $50 \%$ (15 piezas) de la muestra proviene de S1, que está a $350 \mathrm{~km}$ lineales de $\mathrm{El}$ Manso y El Hoyo y a $230 \mathrm{~km}$ de Cholila. Obsidianas de esta fuente están presentes en casi todos los sitios de las tres áreas (Tabla 5 y Figura 3). Esta fuente, ubicada en los alrededores de Cerro Guacho, en la meseta de Somuncurá (Stern et al. 2000), es una de las más ampliamente utilizadas en el norte y centro patagónico (provincias de Neuquén, Río Negro y Chubut y en la zona de Cisnes, en la XI Región de Chile), tal como lo detallan numerosos trabajos (Bellelli et al. 2006; Boschín y Massaferro 2014; Castro Esnal y Stern 2015; Castro Esnal et al. 2011; Favier Dubois et al. 2009; Gómez Otero y Stern 2005; Méndez et al. 2017; Pérez de Micou y Castro 2007; Stern et al. 2000; Stern et al. 2007; Stern et al. 2013).

En los últimos años se han identificado artefactos arqueológicos provenientes de PC1 en diversos sitios de Neuquén, Río Negro y Chubut (Bellelli et al. 2006; Fernández y Vítores 2015; López et al. 2009; Stern et al. 2012; Pérez et al. 2015). También se la ubicó en un sitio del actual territorio chileno, a escasos $70 \mathrm{~km}$ de la fuente (Stern et al. 2009). En esta oportunidad PC1 se identificó en Población Anticura (El Manso), a aproximadamente $370 \mathrm{~km}$ lineales de la fuente (Tabla 5 y Figura 3).

La tercera fuente de la que ya conocíamos su uso en estos ambientes de bosque es Laguna La Larga (LL) que está presente sólo en Cholila, en los tres sitios analizados en este trabajo. Probablemente esto se deba a su cercanía (50 km lineales). Además, hasta el momento parece ser una fuente de uso muy esporádico, tal vez debido a su baja calidad para la talla y a la dificultad de hallar fragmentos de buena calidad en la fuente (Tabla 5 y Figura 3).

Por otro lado, la fuente de Telsen, muy utilizada en sitios del norte de Patagonia (costa, interior de Chubut, y una variedad en río Cisnes (Fabier Dubois et al. 2009; Mendez et al. 2008-2009; 2017; Stern et al. 2000), llamativamente no ha sido registrada en ningún sitio del bosque de Norpatagonia, lo que puede relacionarse con la idea de la circulación de estas rocas en sentido N-S a través del río Chico y hacia la costa (Castro Esnal et al. 2011; Stern et al. 2013). Otras fuentes ausentes en los sitios estudiados son Chaitén y Angostura Blanca. La ausencia de la primera tal vez refleje ciertas restricciones al tránsito de obsidianas a través de la cordillera y el bosque siempre-verde en esta latitud, corroborando lo ya observado en otros trabajos (Bellelli et al. 2008). En cambio el uso de obsidianas de la fuente de Angostura Blanca fue registrado recientemente en el valle del río Cisnes (Méndez et al. 2017) dando cuenta de un tránsito en el eje noreste-suroeste, a través de un ambiente de estepa, que no se vería dificultado por posibles barreras biogeográficas como el bosque siempre-verde o la quebrada topografía que caracteriza a los sectores del norte de Patagonia (Méndez y Reyes 2008).

Estos datos repiten y fortalecen las tendencias que observamos en trabajos anteriores (Bellelli y Pereyra 2002; Bellelli et al. 2006). Los resultados que presentamos en esta oportunidad señalan que en los sitios de El Manso y de Cholila se usaron también obsidianas de fuentes ubicadas en la zona boscosa de los Lagos Lácar y Lolog y en el Cordón Chapelco (provincia de Neuquén), además de otras dos provenientes de algún lugar de la meseta de Somuncurá y de otra no identificada.

Finalmente, las obsidianas analizadas para este trabajo provienen de dos de los tres ambientes volcánicos descritos más arriba. A partir de nuestros resultados, por un lado, se fortalecen las evidencias ya conocidas sobre la utilización de obsidianas de ambientes relacionados con los centros Miocénicos altamente alcalinos asociados con las lavas de retro-arco de la meseta Patagónica (Sacanana, Cerro Castillo y Meseta de Somuncurá) y por otra parte, se suman datos sobre el aprovechamiento de los centros volcánicos Plioceno-Pleistocénicos ligeramente alcalinos del este de los Andes altos (Portada Covunco, Cerro de las Planicies/Lolog, Arroyo Quilahuinto y Arroyo Pocahullo, Paillakura, Yuco y Laguna La Larga) de los que se tenía poca información.

\section{Discusión}

La nueva información generada, sumada a los datos ya disponibles, puede tratarse a partir de dos ejes: el espacial y el cronológico. En primer lugar, se corroboran las tendencias observadas en los trabajos previos en cuanto a que se priorizaron fuentes distantes (en todos los casos, más de $200 \mathrm{~km}$ lineales) pero muy abundantes y de muy buena calidad, en detrimento de la más cercana, Laguna La Larga, que sólo está 


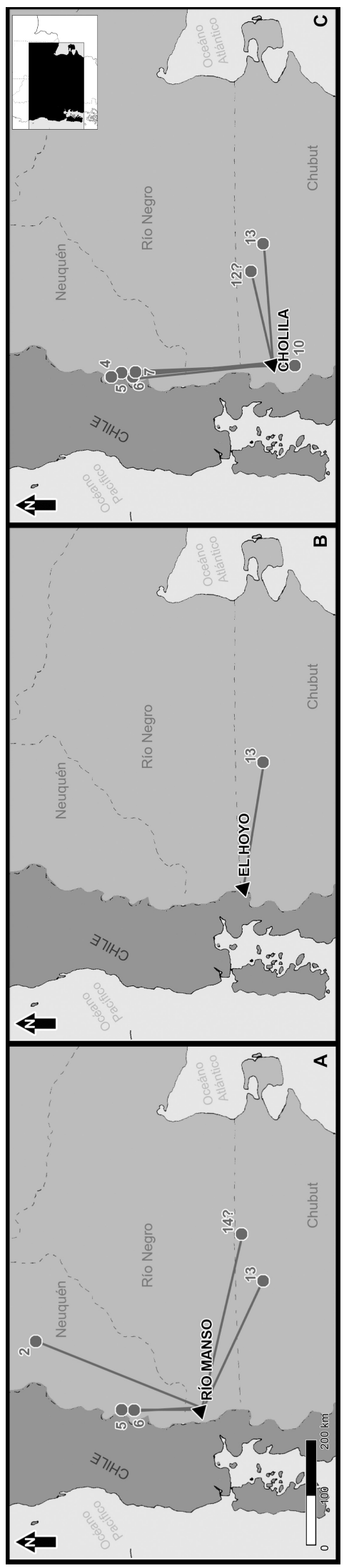

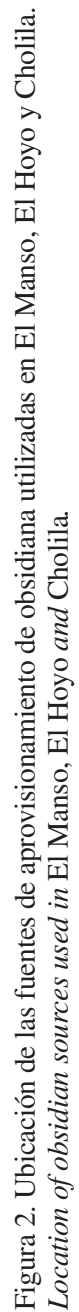


Tabla 3. Elementos traza expresados en $\mu \mathrm{g} \mathrm{g}^{-1}$ Trace elements expressed in $\mu g g^{-1}$.

\begin{tabular}{|c|c|c|c|c|c|c|c|c|c|c|c|c|c|c|}
\hline Localidad & Muestra & $\mathrm{Ti}$ & $\mathrm{Mn}$ & $\mathrm{Rb}$ & $\mathrm{Sr}$ & $\mathrm{Y}$ & $\mathrm{Zr}$ & $\mathrm{Nb}$ & Cs & $\mathrm{Ba}$ & Hf & $\mathrm{Pb}$ & Th & $\mathrm{U}$ \\
\hline \multirow{9}{*}{ El Manso } & PA-1 & 1516 & 599 & 144 & 78 & 27 & 283 & 22 & 5,4 & 479 & 7,8 & 21,9 & 18,0 & 4,7 \\
\hline & PA-2 & 1020 & 360 & 281 & 4 & 50 & 362 & 138 & 3,8 & 18 & 13,0 & 22,7 & 23,1 & 6,3 \\
\hline & PA-3 & 892 & 291 & 350 & 7 & 62 & 439 & 152 & 5,0 & 13 & 16,8 & 34,1 & 24,9 & 7,9 \\
\hline & PA-6 & 1067 & 407 & 162 & 48 & 18 & 167 & 37 & 7,4 & 248 & 11,6 & 19,9 & 32,5 & 7,7 \\
\hline & PL-1 & 958 & 329 & 280 & 7 & 50 & 345 & 138 & 4,0 & 21 & 13,7 & 23,5 & 24,1 & 6,8 \\
\hline & PL-2 & 1428 & 647 & 120 & 178 & 17 & 167 & 18 & 5,1 & 891 & 4,8 & 23,0 & 18,4 & 4,5 \\
\hline & PL-3 & 1007 & 349 & 287 & 6 & 53 & 365 & 144 & 4,0 & 16 & 14,4 & 24,9 & 24,3 & 7,3 \\
\hline & PL-4 & 970 & 324 & 292 & 5 & 52 & 363 & 141 & 4,1 & 15 & 14,8 & 24,8 & 24,8 & 7,0 \\
\hline & PL-5 & 1099 & 957 & 369 & 2 & 61 & 520 & 201 & 7,3 & 7 & 20,6 & 37,2 & 46,7 & 17,9 \\
\hline El Hoyo & RA1-1 & 950 & 307 & 378 & 7 & 63 & 383 & 161 & 5,3 & 12 & 16,7 & 34,2 & 27,3 & 8,3 \\
\hline \multirow{22}{*}{ Cholila } & $\mathrm{CP} 1$ & 800 & 996 & 119 & 47 & 20 & 88 & 17 & 4,2 & 629 & 3,8 & 15,0 & 9,3 & 3,4 \\
\hline & $\mathrm{CP} 3$ & 888 & 392 & 248 & 3 & 48 & 330 & 130 & 3,6 & 11 & 11,9 & 19,7 & 20,0 & 6,5 \\
\hline & CP4 & 857 & 373 & 238 & 3 & 45 & 315 & 128 & 3,6 & 10 & 11,8 & 19,7 & 20,1 & 6,2 \\
\hline & CP5 & 866 & 333 & 269 & 5 & 50 & 292 & 142 & 4,9 & 11 & 11,7 & 21,7 & 24,2 & 8,5 \\
\hline & CP6 & 720 & 644 & 116 & 115 & 12 & 59 & 11 & 5,1 & 837 & 2,7 & 16,3 & 13,0 & 4,4 \\
\hline & $\mathrm{CP} 7$ & 959 & 384 & 247 & 3 & 46 & 333 & 128 & 3,9 & 12 & 13,2 & 22,6 & 22,6 & 6,8 \\
\hline & CP8 & 963 & 961 & 118 & 38 & 20 & 89 & 17 & 4,6 & 718 & 3,7 & 18,9 & 10,8 & 3,6 \\
\hline & CP9 & 490 & 582 & 108 & 52 & 32 & 104 & 20 & 4,4 & 816 & 4,2 & 16,8 & 8,9 & 2,5 \\
\hline & CP10 & 952 & 375 & 250 & 3 & 45 & 328 & 139 & 4,0 & 17 & 17,9 & 23,1 & 28,3 & 6,7 \\
\hline & CP11 & 995 & 379 & 262 & 4 & 48 & 339 & 131 & 4,2 & 14 & 13,9 & 24,5 & 24,6 & 7,2 \\
\hline & CP12 & 917 & 921 & 120 & 38 & 20 & 89 & 19 & 4,6 & 685 & 3,7 & 19,0 & 11,1 & 3,6 \\
\hline & CP13 & 969 & 381 & 265 & 3 & 49 & 349 & 134 & 4,1 & 12 & 13,5 & 24,4 & 24,6 & 7,0 \\
\hline & CP14 & 1673 & 688 & 146 & 78 & 28 & 291 & 24 & 5,6 & 510 & 8,2 & 23,3 & 19,5 & 4,8 \\
\hline & CP15 & 963 & 377 & 273 & 7 & 49 & 346 & 137 & 3,9 & 16 & 13,4 & 24,1 & 24,7 & 6,9 \\
\hline & CP16 & 517 & 569 & 113 & 46 & 33 & 105 & 20 & 4,0 & 795 & 4,0 & 16,1 & 8,8 & 2,4 \\
\hline & LG3-1 & 558 & 564 & 114 & 44 & 34 & 107 & 21 & 4,1 & 824 & 4,3 & 16,5 & 9,0 & 2,4 \\
\hline & LG3-2 & 1140 & 353 & 298 & 1 & 59 & 715 & 149 & 4,2 & 5 & 22,2 & 31,0 & 24,4 & 7,5 \\
\hline & LG3-3 & 4267 & 171 & 68 & 30 & 15 & 117 & 17 & 1,3 & 105 & 8,0 & 22,7 & 9,5 & 0,6 \\
\hline & LG3-4 & 1001 & 375 & 283 & 5 & 51 & 363 & 139 & 4,1 & 12 & 14,0 & 24,4 & 25,5 & 7,1 \\
\hline & LG3-5 & 1031 & 934 & 132 & 42 & 22 & 96 & 18 & 4,8 & 728 & 3,9 & 19,7 & 11,3 & 3,7 \\
\hline & LG4-1 & 931 & 890 & 129 & 41 & 22 & 95 & 18 & 4,7 & 707 & 3,7 & 18,9 & 11,2 & 3,8 \\
\hline & LG4-2 & 471 & 503 & 114 & 46 & 33 & 106 & 21 & 4,0 & 783 & 4,0 & 15,7 & 8,5 & 2,3 \\
\hline
\end{tabular}




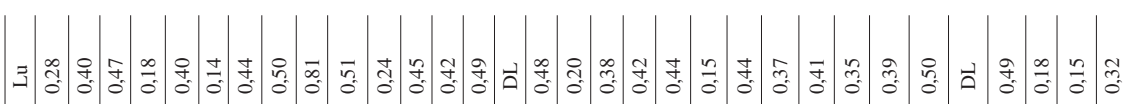

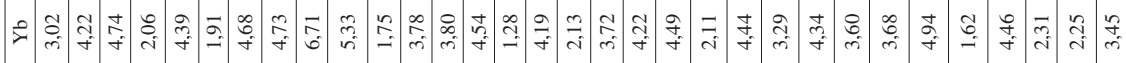

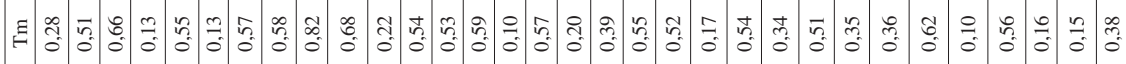

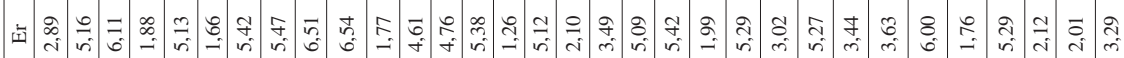

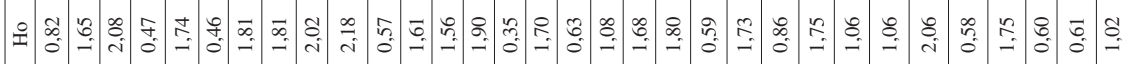

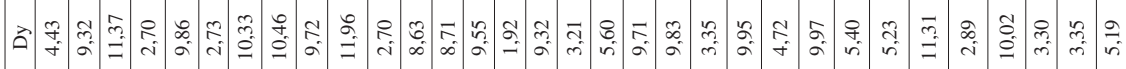

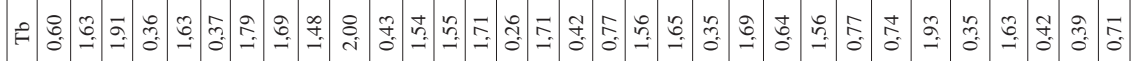

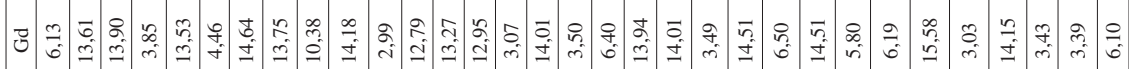

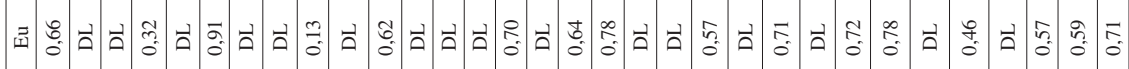

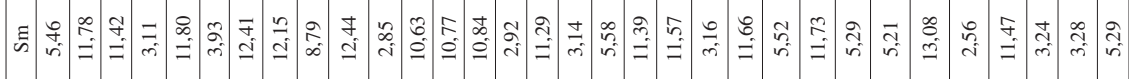

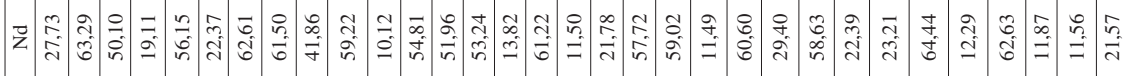

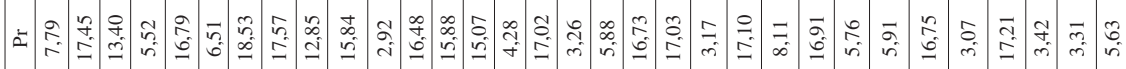

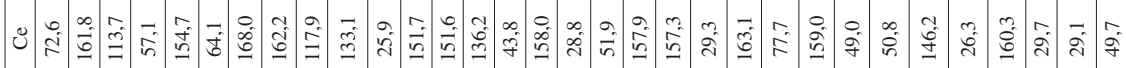

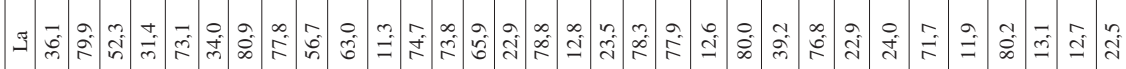

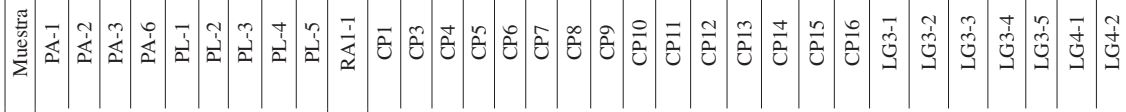

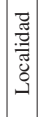

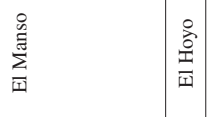


Tabla 5. Procedencia de las obsidianas analizadas Provenance of analyzed obsidians.

\begin{tabular}{|c|c|c|c|}
\hline Fuente/Ambiente & Localidad & Sitio & Muestra \\
\hline Portada Covunco (PC)/estepa & El Manso & Población Anticura (PA) & PA-6 \\
\hline Cerro de las Planicies/Lago Lolog (CP/LL1)/bosque mixto de Nothofagus y Austrocedrus & Cholila & $\begin{array}{l}\text { Los Guanacos } 3 \text { (LG3) } \\
\text { Los Guanacos } 4 \text { (LG4) } \\
\text { Cerro Pintado (CP) } \\
\text { Cerro Pintado (CP) }\end{array}$ & $\begin{array}{c}\text { LG3-5 } \\
\text { LG4-1 } \\
\text { CP-8 } \\
\text { CP-12 }\end{array}$ \\
\hline Arroyo Quilahuinto/Arroyo Pocahullo (QU/AP)/bosque mixto de Nothofagus y Austrocedrus & El Manso & Paredón Lanfré (PL) & PL-2 \\
\hline Yuco (YC)/bosque mixto de Nothofagus y Austrocedrus & Cholila & Cerro Pintado (CP) & $\mathrm{CP}-6$ \\
\hline Paillakura (PK)/bosque mixto de Nothofagus y Austrocedrus & $\begin{array}{l}\text { El Manso } \\
\text { Cholila }\end{array}$ & $\begin{array}{l}\text { Población Anticura (PA) } \\
\text { Cerro Pintado (CP) }\end{array}$ & $\begin{array}{l}\text { PA-1 } \\
\text { CP-14 }\end{array}$ \\
\hline Laguna La Larga (LL)/bosque mixto de Nothofagus y Austrocedrus & Cholila & $\begin{array}{l}\text { Los Guanacos } 3 \text { (LG3) } \\
\text { Los Guanacos } 4 \text { (LG4) } \\
\text { Cerro Pintado (CP) } \\
\text { Cerro Pintado (CP) }\end{array}$ & $\begin{array}{l}\text { LG3-1 } \\
\text { LG4-2 } \\
\text { CP-9 } \\
\text { CP-16 }\end{array}$ \\
\hline Cerro Castillo ? (CC)*/estepa & Cholila & Los Guanacos 3 (LG3) & LG3-2 \\
\hline \multirow[t]{3}{*}{ Sacanana 1 (S1)/estepa } & Cholila & $\begin{array}{l}\text { Cerro Pintado (CP) } \\
\text { Cerro Pintado (CP) } \\
\text { Cerro Pintado (CP) } \\
\text { Cerro Pintado (CP) } \\
\text { Cerro Pintado (CP) } \\
\text { Cerro Pintado (CP) } \\
\text { Cerro Pintado (CP) } \\
\text { Cerro Pintado (CP) } \\
\text { Los Guanacos } 3 \text { (LG3) }\end{array}$ & $\begin{array}{l}\text { CP-3 } \\
\text { CP-4 } \\
\text { CP-5 } \\
\text { CP-7 } \\
\text { CP-10 } \\
\text { CP-11 } \\
\text { CP-13 } \\
\text { CP-15 } \\
\text { LG3-4 }\end{array}$ \\
\hline & El Manso & $\begin{array}{l}\text { Paredón Lanfré (PL) } \\
\text { Paredón Lanfré (PL) } \\
\text { Paredón Lanfré (PL) } \\
\text { Población Anticura (PA) }\end{array}$ & $\begin{array}{l}\text { PL-2 } \\
\text { PL-3 } \\
\text { PL-4 } \\
\text { PA-2 }\end{array}$ \\
\hline & El Hoyo & Risco de Azocar 1 (RA1) & RA1-1 \\
\hline Meseta Somuncurá 1 (MS1)*/estepa & El Manso & $\begin{array}{l}\text { Población Anticura (PA) } \\
\text { Paredón Lanfré (PL) }\end{array}$ & $\begin{array}{l}\text { PA-3 } \\
\text { PL-5 }\end{array}$ \\
\hline Desconocida & Cholila & Los Guanacos 3 (LG3) & LG3-3 \\
\hline
\end{tabular}

* Sin localización conocida.

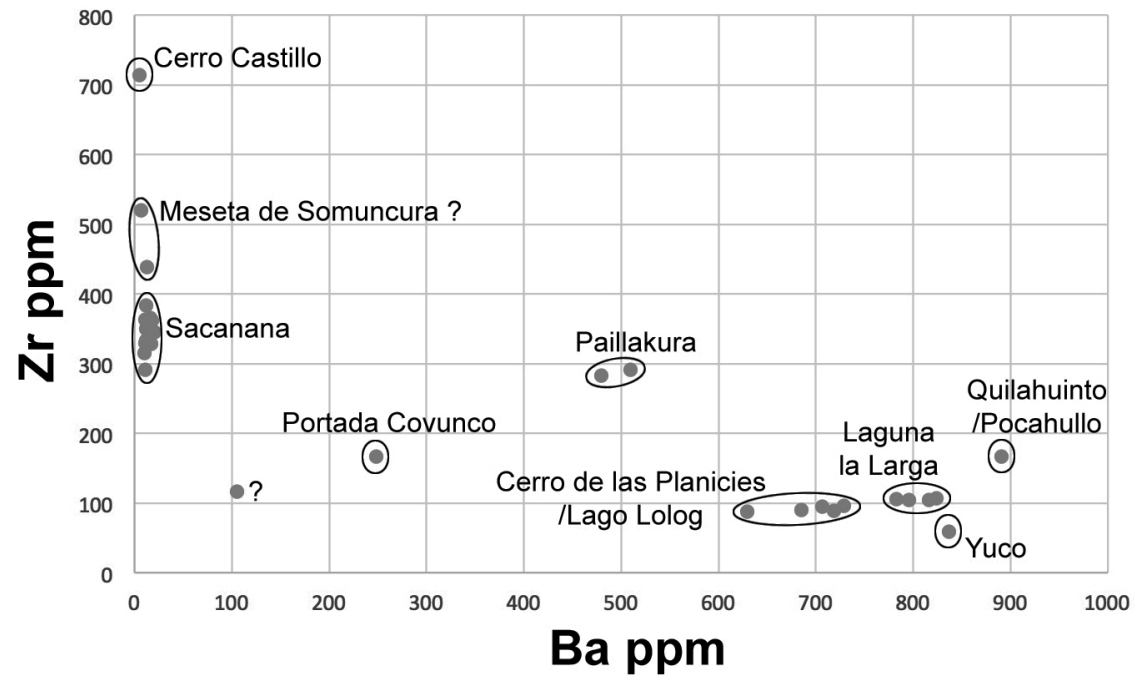

Figura 3. Contenido de Bario vs Zirconio (en partes por millón) para artefactos de obsidiana de los sitios de El Manso, El Hoyo y Cholila comparadas con las elipses de las composiciones de obsidianas de diferentes fuentes conocidas.

Barium versus Zirconium content (in part-per-million) of obsidian artifacts from sites from $\mathrm{El}$ Manso, El Hoyo and Cholila compared to known fields for various possible sources in this region. 
presente en sitios de Cholila y que sigue siendo poco utilizada, debido tal vez a su mala calidad para la talla y/o a la dificultad para el acceso ya que está ubicada en el interior del bosque. Sacanana 1 continúa siendo la más utilizada en las tres regiones y Portada Covunco vuelve a ser identificada pero esta vez asociada a sitios de El Manso, uniéndose a los resultados anteriores de Cholila (Bellelli et al. 2006).

A las tendencias ya conocidas se suman tres elementos novedosos. Por un lado el uso de obsidianas semejantes a las identificadas como Cerro Castillo (en un solo sitio de superficie), que es un tipo conocido hasta ahora sólo a través del análisis de artefactos, desconociéndose su fuente. En segundo término, la utilización en dos sitios de El Manso de obsidianas determinadas como Meseta de Somuncurá 1 , que tampoco ha sido ubicada fehacientemente pero que probablemente proviene de algún sector del interior de dicha meseta. Finalmente, se incorporó el uso de fuentes cercanas a los Lagos Lacar y Lolog, en el bosque mixto de Neuquén (Cerro de las Planicies/Lago Lolog, Yuco, Paillakura, Quilahuinto/ Pocahullo), a $150 \mathrm{~km}$ del valle de El Manso y a $260 \mathrm{~km}$ de Cholila. Este escenario fortalece la idea de una circulación de obsidianas en sentido E-O, conectando la estepa y el bosque y reforzando las propuestas de complementariedad entre ambos ambientes. Al mismo tiempo, la circulación de estas rocas en sentido N-S a través del bosque expone una dimensión diferente en el movimiento de estas materias primas, mostrando una imagen alternativa a la idea tradicional de la complementariedad estepabosque, tal vez, vinculada con un uso más continuo de este último ambiente.

Por otra parte, la baja frecuencia en el uso de obsidianas y el tamaño de los artefactos están en consonancia con los modelos de transporte de materias primas a largas distancias: artefactos terminados, muy conservados o formas base, ausencia de transporte de nódulos o núcleos de tamaño importante (Kuhn 1994, 2004; Roth y Dibble 1998). La distancia influye en la elección de qué transportar y también en la maximización en el aprovechamiento de la obsidiana en los sitios, donde se registran tareas de formatización de filos y/o mantenimiento de artefactos y/o filos y el descarte de los instrumentos al final de su vida útil (Carballido Calatayud 2009). Esta tendencia que se observa en la obsidiana se replica en las otras materias primas de buena calidad registradas en todos los sitios. Se trata de rocas silíceas y calcedonias cuyas fuentes no han sido ubicadas localmente; no sucede lo mismo con las rocas volcánicas (basalto y andesita principalmente) que se registran en baja frecuencia en los sitios y que son de tamaños más grandes que las obsidianas, sílices y calcedonias (Carballido Calatayud 2009; Carballido Calatayud y Pereyra 2012).

A pesar de la baja frecuencia y de las características del uso de la obsidiana, fue posible detectar la utilización de al menos siete fuentes distintas en cinco sitios de Cholila y cuatro fuentes en dos sitios de El Manso, reforzando la idea de fuertes lazos entre ambientes diferenciados y distantes, sobre todo con posterioridad al 2.000 a.p. En cuanto al eje cronológico, la información con que contábamos hasta este momento en el bosque del noroeste de Patagonia indicaba que la utilización de obsidiana estaba restringida al Holoceno Tardío. En nuestra área se remontaba a los últimos 2000 años (Bellelli et al 2006) mientras que en otras alcanza los 3500 aproximadamente (Castro Esnal et al. 2011). Evidencias de uso de obsidiana más temprana se han registrado sólo en unos pocos sitios de la estepa y el ecotono, como Traful 1, Epullán Grande y Cerro Amigo (Fernández y Vítores 2015).

En este trabajo incorporamos el resultado de una muestra proveniente de la fuente MS1 de la Meseta de Somuncurá, recuperada en el nivel más profundo del sitio Población Anticura fechado en 8.230 años a.p., que permite extender la cronología del uso de obsidiana en el bosque al Holoceno Temprano. En los $50 \mathrm{~cm}$ basales de la secuencia, donde obtuvimos cinco fechados que abarcan entre los 7.240 \pm 90 a.p. LP-2727 y $8.230 \pm 110$ a.p. LP-2758, la representación de esta roca es un $11,27 \%$ de los desechos de talla (no se recuperaron instrumentos ni núcleos), porcentaje similar a los de todos los sitios estudiados y a lo visto para el Holoceno Tardío (Bellelli et al. 2013; Podestá et al. 2007). Tampoco hay diferencias respecto del tamaño de esos desechos, siendo todos muy pequeños, tan es así que sólo pudo analizarse uno, ya que el resto no alcanzó el peso mínimo requerido por el método. Esta regularidad que se observa en el uso de la obsidiana a lo largo de todo el Holoceno posiblemente esté vinculada con una necesidad por parte de los grupos humanos de asegurar la disponibilidad de materia prima de excelente calidad en un ambiente en el que esta no está presente o es muy difícil de localizar. Esta tendencia también se observa en las otras materias primas de buena calidad (rocas silíceas y calcedonia).

Hasta el momento el uso de MS1 estaba restringido a sitios de la costa rionegrina y chubutense, de la meseta de Somuncurá, de Maquinchao y en Casa de Piedra de Ortega (Alberti et al 2016; Boschín y Massaferro 2014; Favier Dubois et al. 2009; Fernández y Vitores 2015; Gómez Otero y Stern 2005). En los casos en los que pudieron obtenerse fechados, todos son posteriores a 3.000 a.p. (Boschín y Massaferro 2014; 
Favier Dubois et al. 2009; Fernández y Vitores 2015; Gómez Otero y Stern 2005) por lo que aquí tenemos un dato que lleva al uso de obsidiana de la meseta de Somuncurá en el interior del bosque, al Holoceno Temprano y su traslado a más de $350 \mathrm{~km}$.

\section{Consideraciones Finales}

Este trabajo aporta nueva información para comprender la circulación de la obsidiana tanto a lo largo del espacio como del tiempo en el noroeste de Patagonia. Los resultados de estos análisis corroboraron las tendencias ya conocidas y, además, aportan datos que muestran una ampliación de la cantidad de fuentes aprovechadas en cada área, todas ellas de muy buena calidad y de importancia regional que proveyeron a la mayoría de los sitios conocidos de Norpatagonia. La particularidad de Cholila y de El Manso es que en cada uno de sus sitios se reconocieron obsidianas de muchas fuentes distintas tanto del ámbito boscoso como estepario. Esto podría ser un indicio del conocimiento del espacio regional amplio (Norpatagonia), de sus recursos y de sus vías de circulación en el Holoceno Tardío. Es posible pensar entonces en la existencia de redes centradas en el compartir y/o intercambiar información y materialidades a nivel regional donde las fuentes operarían como centros de distribución recurrentemente utilizados. Se visualizan algunas vías de contacto entre distintas zonas, por ejemplo, en el eje O-E S1, a la que se agregan MS1 y CC, muestran que entre el bosque y la estepa existió una vinculación importante, fortaleciendo la propuesta del uso complementario de recursos de ambos ambientes. La idea de una circulación entre bosque y estepa en el Holoceno Tardío está sustentada por la abundante información disponible 4 . En cambio, para los momentos más tempranos, en el interior del bosque, no se contaba con datos sobre uso de obsidiana hasta la determinación de un desecho de talla de un sitio de este ambiente en El Manso como proveniente de la Meseta de Somuncurá (MS1). De esta manera comienza a perfilarse que ambos ambientes estaban vinculados desde el Holoceno Temprano, momento en que comienza a ser poblada esta meseta y se documenta el uso de obsidianas locales a partir del hallazgo de las 112 puntas cola de pescado en el cerro Los Dos Amigos, de las cuales ocho son de obsidiana (seis fueron analizadas por FRX, resultando cinco de una obsidiana local y una desconocida, Miotti et al. 2012).

La propuesta de un eje de circulación N-S a través del bosque había sido planteada inicialmente a partir de dos líneas de evidencia. Por un lado, de la constatación del uso de obsidiana de una fuente ubicada en el bosque (Laguna La Larga) en sitios de Cholila (Bellelli et al. 2006). Por otra parte, el análisis de los conjuntos cerámicos de los últimos 1.400 años recuperados en sitios de El Manso mostró la presencia de piezas con características coincidentes con la cerámica de tipo Pitrén (Bellelli y Lange 2014). Esta información extiende al sur la presencia de este tipo cerámico que había sido identificado en sitios situados en ambiente de bosque en la zona del lago Traful (Aldazábal et al. 2008-2009), conectando a nuestra área de investigación con sectores más septentrionales del bosque patagónico. $\mathrm{El}$ abundante uso de cuatro fuentes de obsidiana del ámbito boscoso neuquino (CP/LL1; YC; QU/AP y PK) refuerza esta idea de circulación N-S a través de este ambiente en el Holoceno Tardío. La propuesta de un uso recurrente o más intenso del bosque para este momento (Fernández et al. 2013) encuentra en estos resultados y lo planteado por el modelo local que propone el uso recurrente o más intenso del bosque en el tramo final del Holoceno Tardío.

Agradecimientos: Un profundo agradecimiento a los tres evaluadores del trabajo, quienes muy generosamente aportaron ideas que nos ayudaron a mejorar muchos puntos del trabajo original. Estamos en deuda con Ana Forlano, quien realizó los mapas de las Figuras 1 y 2 y con nuestros compañeros de equipo que colaboraron en distintas instancias de este trabajo, desde la recolección de muestras hasta el intercambio y discusión de ideas. También agradecemos a nuestros amigos de El Manso, El Hoyo y Cholila, que nos reciben con hospitalidad y cariño en cada campaña arqueológica. Los trabajos de campo y análisis fueron financiados por la Agencia Nacional de Promoción Científica y Tecnológica (PICT 99762004; 26332-2005; 0471-2012), el Consejo Nacional de Investigaciones Científicas y Tecnológicas (PIP 119/1998; 232/2012; 365/2014), la Universidad de Buenos Aires (UBACyT: U603/2001-2003) y el INAPL (Ministerio de Cultura de la Nación). 


\section{Referencias Citadas}

Alberti, J., M. Cardillo, Ch. Stern y C. Favier Dubois 2016. New results concerning expanded networks of obsidian procurement in San Matías Gulf, Río Negro, Patagonia, Argentina. The Journal of Island and Coastal Archaeology 11:435-442.

Albornoz, A. y A. Hajduk 2006. El área de Nahuel Huapi: 10.000 años de Historia. En Patagonia Total. Antártida e Islas Malvinas, editado por A. Albornoz y A. Hajduk pp. 63-80. Barcel Baires Ediciones, Buenos Aires.

Aldazábal, V., M. Silveira y A. Micaeli 2008-2009. La cerámica del sitio Alero Las Mellizas, Lago Traful, Provincia de Neuquén. Anales de Arqueología y Etnología 63-64:177-195.

Armesto, J.J., P. León Lobos y M. Kalin Arroyo 1995. Los Bosques templados del sur de Chile y Argentina: una isla biogeográfica. En Ecología de los Bosques Nativos de Chile, editado por J.J. Armesto, C. Villagrán y M. Kalin Arroyo, pp. 23-28. Editorial Universitaria, Santiago.

Arrigoni, G. 1997. Pintando entre bosques y lagos. Actas XI Congreso Nacional de Arqueología Argentina. Revista del Museo de Historia Natural de San Rafael Tomo 13:58-63.

Arrigoni, G. 2005. Análisis del territorio de explotación de los recursos básicos para la subsistencia de los grupos prehistóricos que habitaron el valle del Río Desaguadero, Parque Nacional Los Alerces, Chubut. Actas XIII Congreso Nacional de Arqueología Argentina. Tomo 4:63-72. Córdoba.

Arroyo, M.T.K., L. Cavieres, A. Peñaloza, M. Riveros y A.M. Faggi 1995. Relaciones fitogeográficas y patrones regionales de riqueza de especies en la flora del bosque lluvioso templado de Sudamérica. En Ecología de los Bosques Nativos de Chile, editado por J.J. Armesto, C. Villagrán y M. Kalin Arroyo, pp. 71 a 99. Editorial Universitaria, Santiago.

Aschero, C., R. Goñi, T. Civalero, R. Molinari, S. Espinosa, G. Guráieb y C. Bellelli 2005. Holocenic park: Arqueología del Parque Nacional Perito Moreno. En Anales de Parques Nacionales XVII:71-119.Administración de Parques Nacionales, Buenos Aires.

Belardi, J.B. y P. Campan 1999. Estepa y bosque: la utilización de lagos y lagunas en la región de Lago Argentino, Provincia de Santa Cruz. Soplando en el Viento. Actas de las III Jornadas de Arqueología de la Patagonia, pp. 25-41. Facultad de Humanidades, Universidad Nacional del Comahue, Neuquén.

Bellelli, C., M. Carballido Calatayud y P.M. Fernández 2013. Ocupaciones tempranas del bosque norpatagónico: el sitio Población Anticura (valle del Río Manso Inferior, Río Negro). En Actas XVIII Congreso Nacional de Arqueología Argentina, pp. 506. Universidad Nacional de La Rioja.

Bellelli, C., M. Carballido, P. Fernández y V. Scheinsohn 2003. El pasado entre las hojas. Nueva información arqueológica del noroeste de la provincia del Chubut. Argentina. Werken 4:23-42.

Bellelli, C., M. Carballido Calatayud, P. M. Fernández y V. Scheinsohn 2007. Investigaciones arqueológicas en el valle del río Manso Inferior (Pcia. de Río Negro). XVI Congreso Nacional de Arqueología Argentina. Pacarina Tomo III:309-314.

Bellelli, C. y V. Lange 2014. Tiestos en el bosque. Hacia una caracterización de los conjuntos cerámicos del valle inferior del Río Manso (Provincia de Río Negro). Ponencia presentada en las IX Jornadas de Arqueología de la Patagonia, Coyhaique.
Bellelli, C. y F.X. Pereyra 2002. Análisis geoquímicos de obsidiana: distribución, fuentes y artefactos arqueológicos en el noroeste del Chubut (Patagonia Argentina). Werken 3:99-118.

Bellelli, C., F.X. Pereyra y M. Carballido Calatayud 2006. Obsidian localization and circulation in northwestern Patagonia (Argentina): sources and archaeological record. En Geomaterials in Cultural Heritage, editado por M. Maggetti y B. Messiga, pp. 241-255. Geological Society, Special Publications, Londres.

Bellelli, C., V. Scheinsohn y M.M. Podestá 2008. Arqueología de pasos cordilleranos: un caso de estudio en Patagonia Norte durante el Holoceno Tardío. Boletín del Museo Chileno de Arte Precolombino 13:37-55.

Borrero, L.A. y A.S. Muñoz 1999. Tafonomía en el bosque patagónico. Implicaciones para el estudio de su explotación y uso por poblaciones humanas de cazadores-recolectores. En Soplando en el Viento. Actas de las Terceras Jornadas de Arqueología de la Patagonia, pp. 43-56. INAPL. Universidad Nacional del Comahue, Neuquén-Buenos Aires.

Boschín, M.T. y G.I. Massaferro 2014. La obsidiana: una señal geoarqueológica del alcance de las relaciones sociales en la Patagonia Pre y Post-Hispánica. En Arqueología Precolombina en Cuba y Argentina: Esbozos desde la Periferia, editado por O. Hernández de Lara y A.M. Rocchietti, pp. 227-258. Aspha, Buenos Aires.

Carballido Calatayud, M. 2009. Organización de la Tecnología Lítica en el Bosque de Norpatagonia durante el Holoceno Tardío. Aportes para un Modelo de Uso del Bosque en la Comarca Andina del Paralelo $42^{\circ}$. Tesis para optar por el grado de Doctora Facultad Filosofía y Letras, Universidad de Buenos Aires, Buenos Aires.

Carballido Calatayud, M. y P.M. Fernández 2013. La caza de ungulados en el bosque de Patagonia. Aportes desde la localidad de Cholila (Chubut, Argentina). Relaciones 38:59-82.

Carballido Calatayud, M. y F.X. Pereyra 2012. Determinación de la base regional de recursos líticos del área Río Manso inferior-Foyel (Río Negro). Primeros resultados. Comechingonia 16:287-296.

Castro Esnal, A., C. Pérez de Micou y Ch.R. Stern 2011. Circulación de obsidiana en Chubut, Patagonia Central, Argentina: uso de las materias primas extra-regionales como indicadores de movilidad e interacción entre grupos cazadores recolectores. Revista do Museu de Arqueologia e Etnografia 21:93-102.

Castro Esnal, A. y Ch. Stern 2015. Estudios geoquímicos sobre artefactos arqueológicos de obsidiana de contextos estratigráficos y superficiales en Aldea Beleiro, SO de Chubut (Patagonia, Argentina). Ponencia presentada en el VI Congreso Nacional de Arqueometría, pp. .33-35. Río Cuarto.

Durán, V., M. Giesso, M. Glascock, G. Neme, A. Gil y L. Sanhueza 2004. Estudio de fuentes de aprovisionamiento y redes de distribución de obsidiana durante el Holoceno Tardío en el sur de Mendoza (Argentina). Estudios Atacameños 28:25-43.

Espinosa, S.L. 2000. Los conjuntos artefactuales líticos de la estepa y el bosque en el Parque Nacional Perito Moreno (Santa Cruz, Argentina). En Desde el País de los Gigantes. Perspectivas Arqueológicas en Patagonia, pp. 357-367. Universidad Nacional de la Patagonia Austral, Río Gallegos. 
Espinosa, S.L. 2002. Estrategias Tecnológicas Líticas y Uso del Espacio en Momentos Tardíos en el Parque Nacional Perito Moreno (Santa Cruz). Tesis para optar al grado de Doctor en Filosofía y Letras. Facultad de Filosofía y Letras, Universidad de Buenos Aires, Buenos Aires.

Faggi, A.M. 1994. Relevamiento de las comunidades vegetales del Parque Nacional y Reserva Estricta Lago Puelo. Informe final presentado al Centro de Estudios Farmacológicos y Botánicos. Consejo Nacional de Investigaciones Científicas y Técnicas. Ms en poder del autor.

Favier Dubois, C.M., Ch. Stern y M. Cardillo 2009. Caracterización de la distribución y tipos de obsidiana presentes en la costa rionegrina. En Arqueología de Patagonia: Una Mirada desde el Último Confín, editado por M. Salemme, F. Santiago, M. Álvarez, E. Piana, M. Vázquez y E. Mansur, pp. 349-359. Editorial Utopías, Ushuaia.

Fernández, M. y M. Vítores 2015. Aprovisionamiento y uso de obsidianas en la cuenca del río Limay (Provincias de Río Negro y del Neuquén). En Arqueometría Argentina, editado por A. Pifferetti e I. Dosztal, pp. 285-296. Aspha, Buenos Aires.

Fernández, P.M., M. Carballido Calatayud, C. Bellelli y M. Podestá 2013. Tiempo de cazadores. Cronología de las ocupaciones humanas en el valle del río Manso inferior (Río Negro). En Tendencias Teórico-Metodológicas y Casos de Estudio en la Arqueología de la Patagonia, editado por A. F. Zangrando, R. Barberena, A. Gil, G. Neme, M. Giardina, L. Luna, C. Otaola, S. Paulides, L. Salgán y A. Tivoli, pp. 167-175. Museo de Historia Natural de San Rafael, Mendoza.

Gómez Otero, J. y Ch. Stern 2005. Circulación, intercambio y uso de obsidianas en la costa de la provincia del Chubut (Patagonia, Argentina) durante el Holoceno tardío. Intersecciones 6:93-108.

Goñi, R. 1988. Arqueología de momentos tardíos en el Parque Nacional Perito Moreno (Santa Cruz, Argentina). Precirculados de las ponencias científicas presentadas a los simposios del $I X$ Congreso Nacional de Arqueología Argentina pp. 140-151. Buenos Aires.

Hajduk, A., A. Albornoz y M.J. Lezcano 2004. El Mylodon en el patio de atrás. Informe preliminar sobre los trabajos en el sitio El Trébol, ejido urbano de San Carlos de Bariloche, provincia de Río Negro. En Contra Viento y Marea. Arqueología de Patagonia, editado por M.T. Civalero, P.M. Fernández y A.G. Guráieb, pp. 715-731. INAPL-SAA, Buenos Aires.

Hajduk, A., A. Albornoz y M. J. Lezcano 2006. Levels with extinct fauna in the Forest Rockshelter El Trébol (North Patagonia, Argentina). Current Research in the Pleistocene 23:55-57.

Hajduk, A., A. Albornoz y M.J. Lezcano 2011. Espacio, cultura y tiempo: el corredor bioceánico norpatagónico desde la perspectiva arqueológica. En Cultura y Espacio. Araucanía-Norpatagonia, compilado por P. Navarro Floria y W. Delrio, pp. 262-292. Instituto de Investigaciones en Diversidad Cultural y Procesos de Cambio, Universidad Nacional de Río Negro, Bariloche.

Kuhn, S.L. 1994. A formal approach to the design and assembly of mobile toolkits. American Antiquity 59:426-442.

Khun, S.L. 2004. Evolutionary Perspectives on Technology and Technological Change. World Archaeology 36:561-570.

Lezcano, M.J., A. Hajduk y A.M. Albornoz 2010. El menú a la carta en el bosque ¿entrada o plato principal?: una perspectiva comparada desde la zooarqueología del sitio El Trébol (Parque Nacional Nahuel Huapi, Río Negro). En Zooarqueología a Principios del Siglo XXI, editado por M.A. Gutiérrez, M. De Nigris, P.M. Fernández, M. Giardina, A.F. Gil, A. Izeta, G. Neme y H.D. Yacobaccio, pp. 243-257. Ediciones del Espinillo, Buenos Aires.

López, L.,A. Pérezy Ch. Stern 2009. Fuentes de aprovisionamiento y distribución de obsidianas en la provincia de Neuquén (Noroeste de la Patagonia Argentina). Intersecciones 10:75-88.

López, L., M. Silveira y Ch. Stern 2010. Uso de obsidianas entre los cazadores-recolectores en el bosque del lago Traful, Noroeste de la Patagonia, Argentina. Magallania 38:238-245.

Marconetto, B. 2002. Análisis de los vestigios de combustión de los sitios Aleros Don Santiago y Campo Moncada. En Plantas y Cazadores en Patagonia, editado por C. Pérez de Micou, pp. 3353. Facultad de Filosofía y Letras, UBA, Buenos Aires.

Mendez, C. y O. Reyes 2008. Late Holocene human occupation of the Patagonian forests: a case study in the Cisnes river basin. Antiquity 82:560-570.

Mendez, C., Ch. Stern, A. Nuevo Delaunay, O. Reyes, F. Gutiérrez y F. Mena 2017. Spatial and temporal distributions of exotic and local obsidians in Central Western Patagonia, southernmost South America. Quaternary International. En prensa.

Méndez, C., Ch. Stern y O. Reyes 2008-2009. Transporte de obsidianas a lo largo de los Andes de Patagonia Central (Aisén, Chile). Cazadores-Recolectores del Cono Sur 3:51-68.

Miotti, M., E. Terranova, R. Barberena, D. Hermo, M. Giesso y M.D. Glascock 2012. Geochemical sourcing of obsidian fishtail points: studies for the Somuncurá Plateau (Río Negro, Argentina). En Southbound. Late Pleistocene Peopling of Latin America, editado por L. Miotti, M. Salemme, N. Flegenheimer y T. Goebel, pp. 127-131. Center for the Study of the First Americans, Texas A\&M University, Texas.

Onetto, M. 1987. Arte rupestre del valle de Piedra Parada. Provincia de Chubut. Comunicaciones de las Primeras Jornadas de Arqueología de la Patagonia, pp. 195-200. Dirección de Cultura de Chubut, Rawson.

Pérez, A. 2010. Arqueología del bosque. El registro arqueológico del interior y borde de bosque de Norpatagonia. Actas del XVII Congreso Nacional de Arqueología Chilena Tomo 2, pp. 15151528. Sociedad Chilena de Arqueología, Universidad Austral de Chile, Ediciones Kultrún, Valdivia.

Pérez de Micou, C., C. Bellelli y C. Aschero 1992. Vestigios minerales y vegetales en la determinación del territorio de explotación de un sitio. En Análisis Espacial en la Arqueología Patagónica, editado por L.A. Borrero y J.L. Lanata, pp. 53-82. Editorial Ayllu, Buenos Aires.

Pérez de Micou, C. y A. Castro 2007. Los caminos indígenas. La ruta Gan-Gan/Gastre (Chubut). En Aquí Vivieron. Arqueología y Ambiente en Patagonia, editado por C. Pérez de Micou, S. Burry y M. Trivi de Mandri, pp. 9-28. AAINA, Buenos Aires.

Pérez, A. y M. Smith 2008. Eficiencia depredadora y sistema de asentamiento en el bosque Norpatagónico. El área arqueológica Meliquina (Parque Nacional Lanín, Provincia de Neuquén, República Argentina). Las Ciencias, Revista de la Universidad Maimónides 1:83-94.

Pérez, A.E., M. Giesso y M.D. Glascock 2015. Fuentes de aprovisionamiento y uso de obsidianas del ámbito boscoso y lacustre andino norpatagónico (provincia del Neuquén, Argentina). Intersecciones, Volumen especial 2:17-26. 
Podestá, M., C. Bellelli, V. Scheinsohn, P. Fernández, M. Carballido Calatayud, A. Forlano, P. Marchione, E. Tropea, A. Vasini, J. Alberti, M. Gallo y G. Moscovici 2007. Arqueología del valle del río Epuyén (El Hoyo, Chubut). En Arqueología de Fuego-Patagonia. Levantando Piedras, Desenterrando Huesos... y Develando Arcanos, editado por F. Morello, M. Martinic, A. Prieto y G. Bahamonde, pp. 427-442. Centro de Estudios del Hombre Austral, Universidad de Magallanes, Punta Arenas.

Roth, B. y H.L. Dibble 1998. Production and transport of blanks and tools at the French Middle Paleolithic site of Combe Capelle Bas. American Antiquity 63 (1):47-62.

Scheinsohn, V. 2004. "En el país de los ciegos, el tuerto es rey". Visibilidad arqueológica y paisaje en la localidad Cholila. En Contra Viento y Marea. Arqueología de Patagonia, editado por M.T. Civalero, P. Fernández y A.G. Guráieb, pp.581-590. INAPL-SAA, Buenos Aires.

Silveira, M. 1987. Investigaciones arqueológicas en el área boscosa del Lago Traful (Provincia de Neuquén). En Primera Jornada de Arqueología de la Patagonia. Comunicaciones. Gobierno de la Provincia del Chubut. Serie Humanidades 2:295-302. Rawson.

Silveira M. 1996. Alero Los Cipreses (Provincia del Neuquén, República Argentina). En Arqueología Sólo Patagonia, editado por J. Gómez Otero, pp. 107-118. CENPAT-CONICET, Puerto Madryn.

Silveira, M. 1999. El Alero Lariviére: un sitio en el bosque septentrional andino (Departamento Los Lagos, Provincia del Neuquén, Argentina). Actas de las Terceras Jornadas de Arqueología de la Patagonia, pp. 83-92. INAPL y Universidad Nacional del Comahue. Buenos Aires-Neuquén.

Stern, Ch., C. Bellelli y C. Pérez de Micou 2007. Sources and distribution of geologic and archaeologic samples of obsidian from Piedra Parada area, north-central Chubut, Argentine Patagonia. En Arqueología de Fuego-Patagonia: Levantando Piedras, Desenterrando Huesos...y Develando Arcanos, editado por F. Morello, M. Martinic, A. Prieto y G. Bahamonde, pp. 205-208. CEQUA, Punta Arenas.
Stern, Ch., A. Castro, C. Pérez de Micou, C. Méndez y F. Mena 2013. Circulación de obsidianas en Patagonia CentralSur entre 44 y $46^{\circ} \mathrm{S}$. En Tendencias Teórico-Metodológicas y Casos de Estudio en la Arqueología de la Patagonia, editado por A. F. Zangrando, R. Barberena, A. Gil, G. Neme, M. Giardina, L. Luna, C. Otaola, S. Paulides, L. Salgán y A. Tivoli, pp. 243-250. Museo de Historia Natural, SAA e INAPL, San Rafael, Mendoza.

Stern, Ch. y P.J. Curry 1995. Obsidiana del sitio Pose las Conchillas, Chile. Anales del Instituto de la Patagonia 23:119-124.

Stern, Ch., C. García, X. Navarro y J. Muñoz 2009. Fuentes y distribución de diferentes tipos de obsidiana en sitios arqueológicos del centro sur de Chile (38-44º S). Magallania 37:179-192.

Stern, Ch., J. Gómez Otero y J.B. Belardi 2000. Características químicas, fuentes potenciales y distribución de diferentes tipos de obsidiana en el norte de la Provincia del Chubut, Patagonia Argentina. Anales del Instituto de la Patagonia 28:275-290.

Stern, Ch., X. Navarro y J. Muñoz 2002. Obsidiana gris translucida del volcán Chaitén en los sitios arqueológicos de Quilo (Isla Grande de Chiloé) y Chanchan (X región), Chile, y obsidiana de Mioceno en Chiloé. Anales del Instituto de la Patagonia 30:167-174.

Stern, Ch., X. Navarro H., M. Pino y R. Vega 2008. Nueva fuente de obsidiana en la región de la Araucanía, centro-sur de Chile: química y contexto arqueológico de la obsidiana riolítica negra de los Nevados de Sollipulli. Magallania 36:185-193.

Stern, Ch., I. Pereda y A. Aguerre 2012. Primary and secondary sources of a visually variable but chemically distinct obsidian from west-central Neuquén. Archeometry 54:442-453.

Veblen, T., T. Kitzberger y A. Lara 1992. Disturbance and forest dynamics along a transect from Andean rain forest to Patagonian shrubland. Journal of Vegetation Science 3:507-520.

Veblen, T.T., T. Kitzberger, B. Burns y A.L. Rebertus 1995. Perturbaciones y dinámica de regeneración en bosques andinos del sur de Chile y Argentina. En Ecología de los Bosques Nativos de Chile, editado por J.J. Armesto, C. Villagrán y M. Kalin Arroyo, pp. 169-198. Editorial Universitaria, Santiago.

\section{Notas}

1 La utilización de otros métodos, como Fluorescencia de Rayos X (FRX) posibilitarían ampliar el número de piezas analizadas, ya que, además de no destruir el artefacto, no requiere un peso mínimo. Posiblemente, al poder analizar con estos métodos artefactos formatizados (que hemos preservado en esta oportunidad) y desechos muy livianos, se abrirán nuevas líneas de discusión y se amplíe el número de fuentes que ahora no estamos detectando.

2 Los dos desechos de talla que los análisis geoquímicos determinaron que no eran obsidianas fueron seleccionados porque presentaban características macroscópicas (brillo, color y textura) semejantes al resto de las muestras de obsidiana. Se trataba de rocas silíceas de pasta muy homogénea.
Todas las dataciones radiocarbónicas acá presentadas fueron hechas sobre carbón vegetal.

4 El vínculo entre el bosque y la estepa ha sido propuesto sobre la base de distintas líneas de evidencia, entre ellas el uso de recursos vegetales procedentes del área boscosa recuperados en contextos de estepa en el área de Piedra Parada (Chubut, Marconetto 2002; Pérez de Micou 2002), motivos de manifestaciones rupestres similares en sitios de esta región de Chubut y El Bolsón y El Hoyo (Onetto 1987); la presencia en sitios de bosque de instrumentos confeccionados sobre huesos de animales de la estepa (Fernández com. personal 2017; Silveira 1996; Hajduk et al. 2004) y un patrón decorativo similar entre tiestos cerámicos de contextos de estepa y algunos provenientes de un sitio de Cholila (Bellelli com. personal 2003). 